\title{
Cytological Evaluation of Metastatic Melanoma: A Series of 3 Cases with Review of Literature
}

\author{
Prakash S, Saxena A*, Sanjay A, Gajender G, Goel V and Sen R
}

Department of pathology, PGIMS, Rohtak, Haryana, India

*Corresponding author: Saxena A, Department of pathology, PGIMS, Rohtak, Haryana, India, Tel: 91-8743095585; E-mail: ayushisxn01@gmail.com

Received date: October 08, 2018; Accepted date: October 16, 2018; Published date: October 23, 2018

Copyright: ( 2018 Prakash S, et al. This is an open-access article distributed under the terms of the Creative Commons Attribution License, which permits unrestricted use, distribution, and reproduction in any medium, provided the original author and source are credited.

\begin{abstract}
Malignant melanoma is a dangerous tumor with potential to metastasize. We report a series of 3 cases, which presented with lymphadenopathy and their cytological diagnosis of metastatic melanoma was made based on cytomorphological features. FNAC is a highly accurate, rapid, and cost-effective procedure for the diagnosis of metastatic melanoma and should be considered as the initial diagnostic procedure of choice in patients with melanoma with clinically suspected metastases.
\end{abstract}

Keywords: Fine needle aspiration cytology (FNAC); Lymphadenopathy; Metastatic melanoma; Immunohistochemical

\section{Introduction}

Melanoma is an aggressive tumor, accounting for approximately $3 \%$ of all cancers worldwide. In India, its incidence is less than $0.5 \%[1,2]$. White young and middle aged males are affected more than females. The commonest site in males is the trunk followed by head and neck, whereas in females it is the lower limb [3]. Survival in patients with melanoma is related to the stage of the disease at diagnosis, and patients with metastatic melanoma have much poorer survival than those without metastases [4]. So, early detection of both primary malignant melanoma and metastatic disease is important for initiation of appropriate treatment. As a whole, primary melanomas metastasize most frequently to the regional lymph nodes. Herein, we report a series of three cases in which cytological diagnosis of metastatic melanoma was made prior to histological diagnosis.

\section{Case Report}

Case 1: 35 years male presented with swelling of right inguinal region for 4 months. On examination, a firm, fixed swelling measuring $3 \times 2 \times 1 \mathrm{~cm}$ was noted. Patient was a known operated case of malignant melanoma in right foot.

Case2: 62 years female came with swelling of right inguinal region for 1 month, exanimation revealed firm, tender, fixed swelling measuring $1.5 \times 1 \times 1 \mathrm{~cm}$ in right inguinal region. The patient was already diagnosed with malignant melanoma of vulva on biopsy.

Case 3: 37 years male presented with swelling in axilla since 5 months, examination showed swelling, firm in consistency, fixed to underlying skin measuring $2 \times 1 \times 0.5 \mathrm{~cm}$, subsequently patient presented with ulceration of right thumb which on biopsy revealed malignant melanoma.

On aspiration, all the three lymph nodes yielded blackish material mixed with blood. Cytological examination revealed scattered, groups and sheets of pleomorphic round tumor cells having hyperchromatic large nucleus, prominent nucleoli and abundant basophilic cytoplasm.
Few binucleated forms were also seen in the background of lymphocytes and hemorrhage. Tumor cells also showed black pigment granules in the cytoplasm (Figures 1-3). Masson - Fontana stain was positive which confirmed the melanin pigment (Figure 4). Cell block was prepared from the material aspirated for ancillary studies and tumor cells were subjected to HMB45 and S100. All were positive for both the markers, confirming the diagnosis of melanoma (Figures 5 and 6). All the patients underwent surgical excision of the involved node followed by adjuvant radiotherapy. Biopsy examination of excised lymph nodes confirmed the already made diagnosis. Two of the patients are still under follow-up while one with primary diagnosis of malignant melanoma of vulva expired.

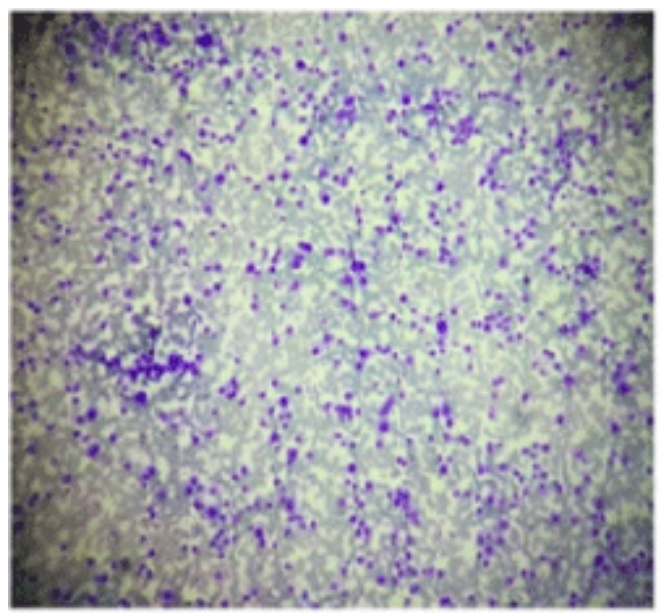

Figure 1: Largely dispersed population of pleomorphic epithilioid cells (Leishman stain, 100k). 
Citation: Prakash S, Saxena A, Sanjay A, Gajender G, Goel V, et al. (2018) Cytological Evaluation of Metastatic Melanoma: A Series of 3 Cases with Review of Literature. J Cytol Histol 9: 522. doi:10.4172/2157-7099.1000522

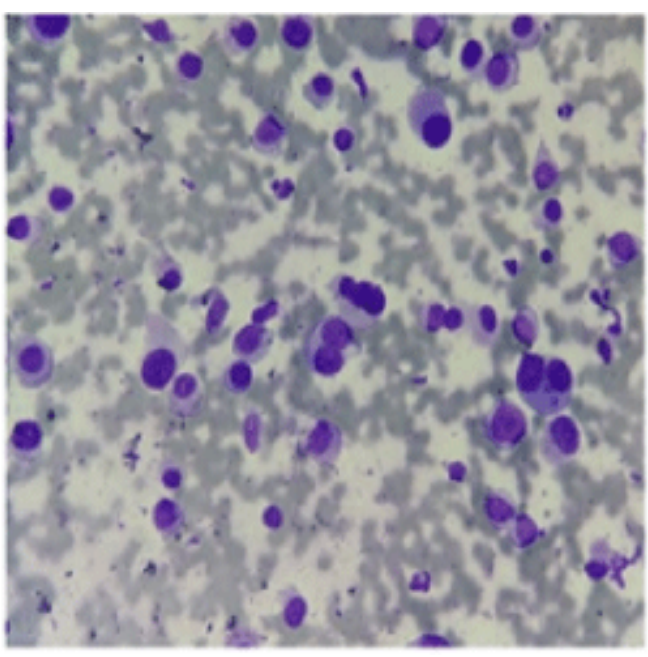

Figure 2: Pleomorphic round tumor cells having hyper chromatic large eccentric nucleus and abundant basophilic cytoplasm (Leishman stain, 400k).

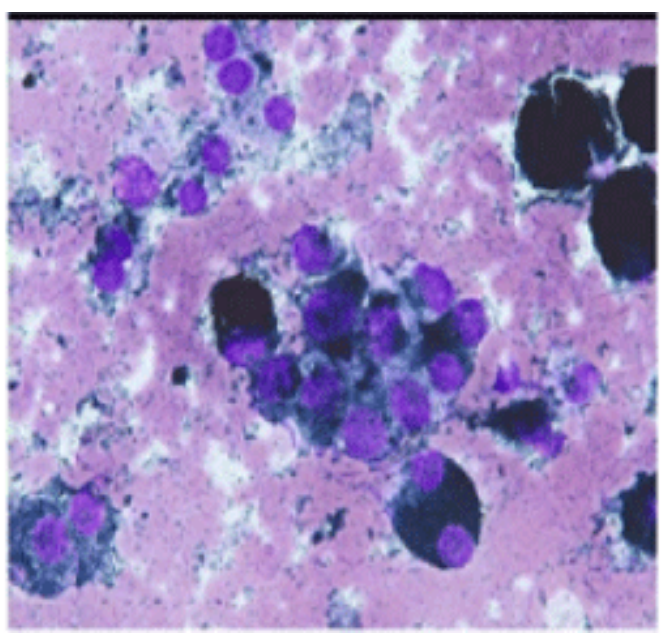

Figure 3: Tumor cells showing bluish black pigment granules in cytoplasm (Leishman, 400k).

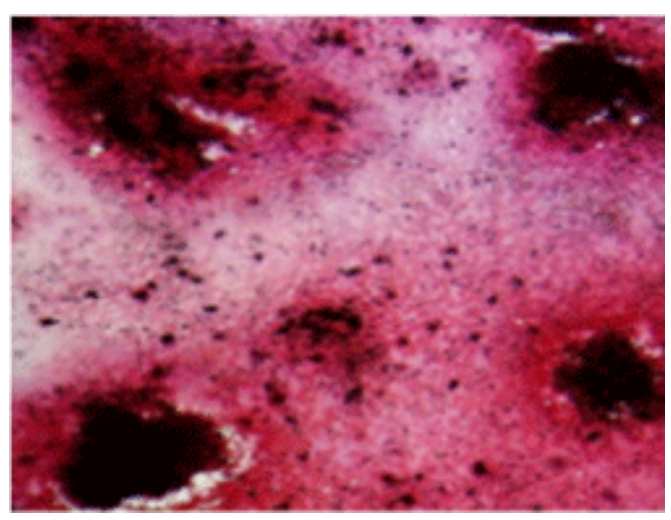

Figure 4: Massons Fontana-special stain: shows blackish colored granules in cytoplasm.

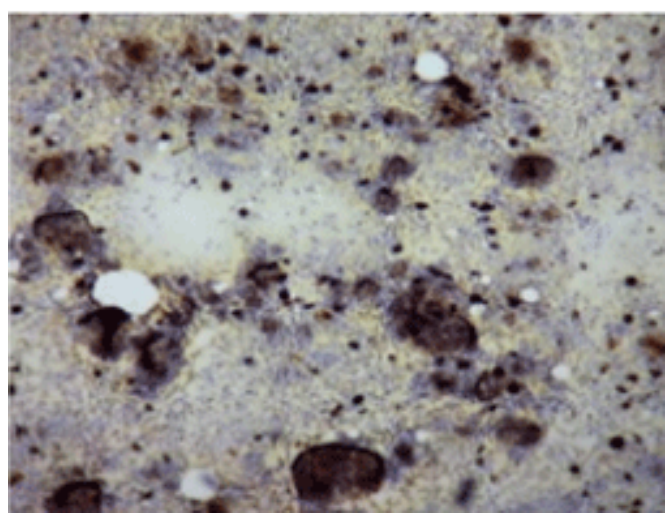

Figure 5: Positivity of HMB45 in tumor cells (HMB45; 400k).

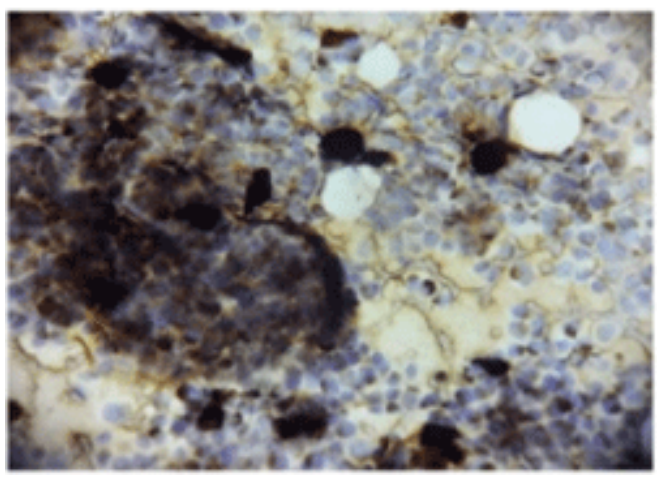

Figure 6: Positivity of S-100 in tumor cells (S-100, 400k).

\section{Discussion}

The incidence of cutaneous melanoma has been increasing annually at a more rapid rate compared to any other type of cancer. Moreover, unlike other solid tumors, melanoma mostly affects young and middleaged individuals (median age at diagnosis $=57$ years). Ultraviolet (UV) 
light radiation from sunlight is the main environmental risk factor for melanoma skin cancer development. The increased risk of melanoma due to sun exposure is directly associated with the UV level and in particular to the UV-B spectrum. In addition, sun exposure patterns and timing have been associated in a number of studies with an increased risk of melanoma. In particular, intense and intermittent sun exposure (typical of sunburn history) is associated with a higher risk compared to a chronic continuous pattern of sun exposure. Up to $90 \%$ of melanomas exhibit an aberrant MAPK pathway activation and this is a central step in melanoma development, being responsible for cell cycle deregulation and apoptosis inhibition. Among the different mechanisms responsible for abnormal MAPK pathway signaling in melanoma, the most frequent genetic abnormalities are BRAF mutations [5].

Cutaneous melanoma is ominous because it is more likely to spread, or metastasize and it usually involves regional lymph nodes first. It is still unclear why lymph node is first site to be involved in metastasis of malignant melanoma. Some studies suggest melanomas express ligands/receptors that would cause preferential homing of melanoma cells to lymph nodes. Chemokine/chemokine receptors and adhesion molecules are potential candidates of such ligand/receptor pairs [6].

Diagnosis of metastaic melanoma is made on FNAC and biopsy. FNAC being rapid, cheap and minimally invasive method of diagnosis is preferred over biopsy, having sensitivity and specificity of $97 \%$ and $99 \%$ respectively [7]. There are 2 major goals in the FNAC diagnosis of metastatic melanoma: (1) to confirm malignancy and exclude benign or reactive processes and (2) to identify the tumor as melanoma and exclude mimickers like dermatopathic lymphadenopathy and tattoo pigment lymphadenopathy. Dermatopathic lymphadenopathy is a distinctive reaction pattern in lymph nodes characterized by paracortical hyperplasia composed of interdigitating dendritic cells, Langerhans cells and macrophages containing melanin pigment. Tattoo pigment lymphadenopathy occurs when macrophages that ingest tattoo pigment migrate through the lymphatic channels to the regional lymph nodes. The nodes with persistent pigment in them become hyperplastic, which may cause a pseudolymphomatous reaction.

FNAC of dermatopathic lymphadenopathy, shows numerous blood vessels on low power, surrounded on either side by large aggregates of histiocytic cells with intervening lymphoid tissue. The cells have abundant, wispy cytoplasm with indistinct cytoplasmic borders. The nuclei are elongated and vesicular, with a fine chromatin pattern and absent or inconspicuous nucleoli, these histiocytes are melanin laden and show positivity for $\mathrm{S} 100$ [8]. It is distinguished from metastatic melanoma, as there is no history of cutaneous primary melanoma in such patients and no cytomorphological features resembling that of melanoma cells. Likewise, in tattoo pigment lymphadenopathy, cytological examination reveals benign histiocytes with carbon deposits and immunohistochemical markers HMB 45 and S100 are negative ruling out metastatic melanoma [9].
In our study, cytological evaluation was carried out for all three lymphadenopthies at various sites, primary being cutaneous melanoma. FNAC yielded blackish material mixed with blood. Cytological examination revealed groups, sheets and singly scattered round tumor cells with pleomorphism containing high $\mathrm{N}: \mathrm{C}$ ratio with prominent nucleoli and abundant basophilic cytoplasm. Few binucleated forms were also seen in the background of lymphocytes and hemorrhage. Tumor cells also showed bluish black pigment granules in cytoplasm suggestive of metastatic malignant melanoma. Masson-Fontana stain was used to confirm the melanin pigment. Cell block was made from material aspirated on which immunohistochemical markers HMB45 (cytoplasmic staining of malignant cells) and S100 were applied to establish our diagnosis which were found to be positive.

Marked improvements in melanoma treatment have been achieved over the past decade. The researchers have shed light on essential mechanisms involved in melanoma biology, thus paving the way for targeted treatment and immunotherapy.

\section{Conclusion}

FNAC is a rapid, safe and cost-effective technique. It gives early and accurate results with minimal invasion thus, saving cost and time to reach to final diagnosis. It is therefore concluded that FNAC is a useful tool in early diagnosis of metastatic lymphadenopathy with good certainty.

\section{References}

1. DeMay R (1996) Gut course. In: DeMay R (ed.) The art and science of cytopathology. ASCP Press, Chicago, IL, pp: 520-521.

2. Boyle P, Maisonneve P, Dore JF (1995) Epidemiology of malignant melanoma. Br Med Bull 51: 54.

3. Weinstock MA (2000) Early detection of melanoma. Jama 284: 886-889.

4. Balch CM, Buzaid AC, Soong SJ, Atkins MB, Cascinelli N, et al. (2001) Final version of the American Joint Committee on Cancer staging system for cutaneous melanoma. J Clin Oncol 19: 3635-3648.

5. Leonardi GC, Falzone L, Salemi R, Zanghì A, Spandidos DA, et al. (2018) Cutaneous melanoma: from pathogenesis to therapy. International journal of oncology 52: 1071-1080.

6. Zbytek B, Carlson JA, Granese J, Ross J, Mihm M, et al. (2008) Current concepts of metastasis in melanoma. Expert Review of Dermatology 3: 569-585.

7. Hafström L, Hugander A, Jonsson PE, Lindberg L-G (1980) Fine-needle aspiration cytodiagnosis of recurrent malignant melanoma. J Surg Oncol 15: 229-234.

8. Iyer VK, Kapila K, Verma K (1998) Fine needle aspiration cytology of dermatopathic lymphadenitis. Actacytologica 42: 1347-1351.

9. Chikkamuniyappa S, Sjuve-Scott R, Yeh IT (2005) Tattoo pigment in sentinel lymph nodes: a mimicker of metastatic malignant melanoma. Journal of Cutaneous Pathology 32: 81-90. 